\title{
Sinoporphyrin sodium is a promising sensitizer for photodynamic and sonodynamic therapy in glioma
}

\author{
YA-WEN AN $^{1,2}$, HAN-QING LIU ${ }^{2,3}$, ZI-QIAN ZHOU ${ }^{3}$, JIAN-CHUN WANG ${ }^{2}$, \\ GUANG-YU JIANG ${ }^{2}$, ZHI-WEN LI ${ }^{2}$, FENG WANG ${ }^{4}$ and HONG-TAO JIN ${ }^{5}$
}

\author{
${ }^{1}$ School of Chemical Biology and Biotechnology, Peking University Shenzhen Graduate School, \\ Shenzhen, Guangdong 518055; ${ }^{2}$ Science and Education Department, Shenzhen Samii Medical Center, \\ Shenzhen, Guangdong 518118; ${ }^{3}$ Peking University Shenzhen Hospital, Shenzhen, Guangdong 518036; \\ ${ }^{4}$ Henan Key Laboratory of Medical Tissue Regeneration, School of Basic Medical Sciences, \\ Xinxiang Medical University, Xinxiang, Henan 453002; ${ }^{5}$ Institute of Materia Medica, \\ Chinese Academy of Medical Sciences and Peking Union Medical College, Beijing 100050, P.R. China
}

Received October 31, 2019; Accepted June 24, 2020

DOI: $10.3892 / o r .2020 .7695$

\begin{abstract}
The aim of the present study was to explore the antitumor effects of sinoporphyrin sodium (DVDMS)-mediated photodynamic therapy (PDT) and sonodynamic therapy (SDT) in glioma, and to reveal the underlying mechanisms. The uptake of DVDMS by U-118 MG cells was detected by flow cytometry (FCM). A 630-nm semiconductor laser and $1-\mathrm{MHz}$ ultrasound were used to perform PDT and SDT, respectively. Cell proliferation and apoptosis were evaluated
\end{abstract}

Correspondence to: Dr Han-Qing Liu, Science and Education Department, Shenzhen Samii Medical Center, 1 Jinniu West Road, Pingshan, Shenzhen, Guangdong 518118, P.R. China

E-mail: liuhanqing@ssmc-sz.com

Dr Feng Wang, Henan Key Laboratory of Medical Tissue Regeneration, School of Basic Medical Sciences, Xinxiang Medical University, 601 Jinsui Road, Hongqi, Xinxiang, Henan 453002, P.R. China

E-mail: wfeng100@126.com

Abbreviations: PDT, photodynamic therapy; SDT, sonodynamic therapy; DVDMS, sinoporphyrin sodium; PBS, phosphate-buffered saline; FCM, flow cytometry; H\&E, hematoxylin and eosin; NAC, N-acetyl-L-cysteine; ROS, reactive oxygen species; PCNA, proliferating cell nuclear antigen; Bcl-2, B-cell lymphoma-2; Bcl-xL, B-cell lymphoma-extra large; Bax, Bcl-2-associated X; p-PI3K, phospho-phosphatidylinositol 3 kinase; p-AKT, phospho-protein kinase B; p-mTOR, phospho-mammalian target of rapamycin; p-p70s6k, phospho-p70 ribosomal s6 kinase; p-rps6, phospho-ribosomal protein s6; p-4EBP1, phospho-4E binding protein 1; p-eIF4E, phospho-protein synthesis initiation factor 4E; SQSTM1/p62, sequestosome 1; LC3, light chain 3; ATCC, American Type Culture Collection; STR, short tandem repeat

Key words: sinoporphyrin sodium, photodynamic therapy, sonodynamic therapy, glioma, protein expression and phosphorylation using the Cell Counting Kit-8 assay, FCM and Hoechst 33258 staining, respectively. Western blot analysis was used to detect protein expression and phosphorylation levels. BALB/c nude mice were used to establish a xenograft model of U-118 MG cells. DVDMS was injected intravenously and PDT and SDT were performed $24 \mathrm{~h}$ later. An in vivo imaging system was used to evaluate the fluorescence of DVDMS, to measure tumor sizes, and to evaluate the therapeutic effects. The uptake of DVDMS by U-118 MG cells was optimal after $4 \mathrm{~h}$. PDT and SDT following DVDMS injection significantly inhibited the proliferation and increased apoptosis of glioma cells in vitro $(\mathrm{P}<0.05, \mathrm{P}<0.01)$ respectively. In vivo, the fluorescence intensity of DVDMS was lower in the PDT and SDT groups compared with the DVDMS group, while tumor cell proliferation and weight were lower in the PDT and SDT groups than in the control group $(\mathrm{P}<0.05, \mathrm{P}<0.01)$. However, there was no significant difference when laser, ultrasound or DVDMS were applied individually, compared with the control group. Hematoxylin and eosin staining suggested that both PDT and SDT induced significant apoptosis and vascular obstruction in cancer tissues. DVDMS-mediated PDT and SDT inhibited the expression levels of proliferating cell nuclear antigen (PCNA) and Bcl-xL, increased cleaved -caspase 3 levels, and decreased the protein phosphorylation of the PI3K/AKT/mTOR signaling pathway. Changes in the expression of PCNA, and Bcl-xL and in the levels of cleaved-caspase 3 were partly reversed by $\mathrm{N}$-acetyl-L-cysteine, a reactive oxygen species (ROS) scavenger. Similar results were obtained with FCM. DVDMS-mediated PDT and SDT inhibited glioma cell proliferation and induced cell apoptosis in vitro and in vivo, potentially by increasing the generation of ROS and affecting protein expression and phosphorylation levels.

\section{Introduction}

Photodynamic therapy (PDT) was first introduced over 100 years ago, and causes cell death through interactions of a photosensitizer, light source of appropriate wavelength, and 
oxygen. PDT is widely used in dermatology, pulmonology, urology, and ophthalmology (1). It is considered as a safe and effective treatment option for several dermatological conditions. Furthermore, the applications of PDT in the treatment of tumors has made great progress in recent years. However, PDT has certain limitations, such as poor penetration of light and the risk of adverse reactions such as pain and burns (2). Therefore, a novel technology termed sonodynamic therapy (SDT) has been developed in recent decades. Similar to PDT, SDT involves sensitizers and oxygen; however, the main difference is that ultrasound, rather than a laser is used as the energy source to activate the sensitizer. Ultrasound is more penetrating than light, has fewer side effects and induces cavitation effects, therefore SDT may have some advantages in treating tumors (3-5).

As new strategies for cancer treatment, PDT and SDT result in fewer side effects, and increased selectivity, adaptability and compliance, compared with traditional methods. Moreover, the combination of PDT/SDT and surgery, chemoradiotherapy and immunotherapy may produce synergistic effects $(6,7)$. However, most of the early generation photosensitizers, such as Photofrin are mixtures produced with no quality control standards (8). Side effects such as pain, burning, and phototoxicity limit the application of these photosensitizers (9). Therefore, there is a requirement for the development of safe and effective sensitizers.

Sinoporphyrin sodium (DVDMS) is a novel photosensitizer isolated from Photofrin derivatives. The activity of DVDMS is similar to that of Photofrin at $10 \%$ of the dosage. DVDMS has good water and lipid solubility, and can be activated by both laser and ultrasound. Preliminary studies have demonstrated that DVDMS is an effective and safe photosensitizer for PDT and inhibits antitumor effects both in vitro and in vivo $(10,11)$. Furthermore, previous studies revealed that DVDMS can achieve therapeutic effects following ultrasound activation $(12,13)$.

Glioma is an intractable primary cancer of the central nervous system that is typically treated with surgery, drugs, and immunotherapy. However, these treatment modalities have certain limitations, and the development of new therapeutic approaches is of great importance to improve patient outcomes (14-17). Recent studies have indicated that PDT and SDT are promising new therapeutic modalities for the treatment of gliomas (18-21). Therefore, the aim of the present study was to evaluate the antitumor effects of DVDMS-PDT and DVDMS-SDT using human brain glioma cells in vitro and a xenograft nude mouse model in vivo. In vivo ultrasound and fluorescence imaging systems were used to evaluate the fluorescence of DVDMS in vivo, tumor size, and therapeutic effects, while FCM, Hoechst 33258 staining and western blot analysis were performed to reveal the potential underlying mechanisms.

\section{Materials and methods}

Reagents. DVDMS, kindly provided by Professor Qi-Cheng Fang (Institute of Materia Medica, Chinese Academy of Medical Sciences and Peking Union Medical College), was dissolved in normal saline to a concentration of $1,000 \mu \mathrm{g} / \mathrm{ml}$, stored at $4^{\circ} \mathrm{C}$ in the dark, and used within 1 month. U-118 MG and U-87 MG human glioma cells were purchased from Procell Life Science \& Technology Co., Ltd. and cultured in
Dulbecco's modified Eagle's medium (Gibco; Thermo Fisher Scientific, Inc.) supplemented with $10 \%$ fetal bovine serum (Gibco; Thermo Fisher Scientific, Inc.).

Animals. A total of 48 BALB/c nude mice (4 weeks old, 16-20 g, 24 male and 24 female), were obtained from Guangdong Medical Laboratory Animal Center (Foshan, Guangdong, China). The animals were housed under a 12-h light/dark cycle at $19.2-25.1^{\circ} \mathrm{C}$ and relative humidity of $42-59 \%$ with ad libitum access to food and water. Animals were allowed to acclimatize to a specific pathogen-free environment for 5 days prior to treatment. The study protocol was approved by the Ethics Committee of Shenzhen PKU-HKUST Medical Center (SYXK 2015-0106) and was performed in accordance with the International Guidelines on the Care and Use of Animals for Scientific Purposes. The animal health and behavior were monitored every day. Any unnecessary operation was prohibited to avoid suffering and distress of the animals.

Measurement of the cellular uptake of DVDMS by U-118 MG cells. Both U-87 MG (cat. no. CL-0238) and U-118 MG (cat. no. CL-0458) cell lines were purchased from Procell Life Science \& Technology Co. Ltd., and the original preservation organization was ATCC. The U-87 MG cell line used in this study was established from glioblastoma of unknown origin. Both the two cell lines were authenticated using STR profiling (authentication reports were provided for the review process).

Absorption spectra of DVDMS were measured using a micro-spectrophotometer (Q5000; Quawell Technology, Inc.). A total of five absorption peaks at 369, 517, 550, 577 and $631 \mathrm{~nm}$, respectively, were observed for DVDMS in PBS. U-118 MG cells were adjusted to a cell density of $1 \times 10^{6}$ cells $/ \mathrm{ml}$ and inoculated $1 \mathrm{ml}$ cell suspension was plated per well in a 6-well plate. A total of $1 \mathrm{ml}$ DVDMS $(10.00 \mu \mathrm{g} / \mathrm{ml})$ was added to each well and the plate was incubated for $0,2,4$, or $6 \mathrm{~h}$. The cells were subsequently collected and washed with PBS. A single cell suspension in PBS was analyzed using an Accuri ${ }^{\text {TM }}$ C6 Plus flow cytometer (BD Biosciences). DVDMS uptake was measured using the FL2 channel $(585 / 40 \mathrm{~nm})$.

Effects of DVDMS-mediated PDT and SDT on cell proliferation and apoptosis. U-118 MG and U-87 MG cells $\left(1 \times 10^{6}\right.$ cells $\left./ \mathrm{ml}\right)$ were inoculated in culture plates for $24 \mathrm{~h}$ and then divided into three groups: DVDMS, PDT and SDT groups. Each group was treated with DVDMS at the same concentration. After incubation for $4 \mathrm{~h}$, cells in the PDT group were exposed to a laser at $630 \mathrm{~nm}$ using a PDT-630 Semiconductor Laser Unit (Guilin Xingda Optoelectronic Medical Instrument Co., Ltd.) with a luminous flux of $50 \mathrm{~mW} / \mathrm{cm}^{2}$ for $10 \mathrm{~min}$ (total energy, $30 \mathrm{~J} / \mathrm{cm}^{2}$ ). Cells in the SDT group were exposed to ultrasound at a frequency of 1.0 MHz using an AFG3022B Ultrasonic Signal Generator (Tektronix Inc.) at an intensity of $500 \mathrm{~mW} / \mathrm{cm}^{2}$ for $1 \mathrm{~min}$ (total energy was $30 \mathrm{~J} / \mathrm{cm}^{2}$ ), as previously described $(11,13)$. Cells in the DVDMS group were not exposed to laser or ultrasound, and served as a control.

Cell proliferation was measured using the Cell Counting Kit (CCK)-8 assay (Dojindo Molecular Technologies, Inc.) at $24 \mathrm{~h}$ after treatment. The concentrations of DVDMS investigated were $0,0.03,0.06,0.13,0.25,0.50,1.00,2.00,4.00$ and $8.00 \mu \mathrm{M}$. After the addition of $10 \mu \mathrm{l} \mathrm{CCK}-8$ reagent to 
$100 \mu \mathrm{l}$ medium, the cells were cultured at $37^{\circ} \mathrm{C}$ for $1 \mathrm{~h}$ and the optical density of the cells was determined at an absorbance of $450 \mathrm{~nm}$ and a reference wavelength of $405 \mathrm{~nm}$ using a Spectra Max i3x Microplate Reader (Molecular Devices).

The Hoechst 33258 Staining kit (cat. no. C0003, Beyotime Institute of Biotechnology) was used to observe the influence of different treatment times on cell apoptosis. Cells were divided into 6 groups: The control, laser, ultrasound, DVDMS, PDT and SDT groups. Cells in the control group were not treated, while cells in the laser and ultrasound groups were exposed to laser $\left(50 \mathrm{~mW} / \mathrm{cm}^{2}\right.$ for $\left.10 \mathrm{~min}\right)$ and ultrasonic radiation $\left(500 \mathrm{~mW} / \mathrm{cm}^{2}\right.$ for $\left.1 \mathrm{~min}\right)$, respectively. For the DVDMS, PDT and SDT groups, the concentration of DVDMS was $0.5 \mu \mathrm{M}$, and cells in PDT and SDT group received laser or ultrasound irradiation aforementioned. At 1, 2, 4, 8 and $24 \mathrm{~h}$ after treatment, $0.5 \mathrm{ml}$ Hoechst 33258 dye solution was used for DNA staining. An IX51 fluorescence microscope (200X, Olympus Corporation) was used to observe the apoptotic cells.

The PE Annexin V Apoptosis Detection kit I (BD Pharmingen ${ }^{\mathrm{TM}}$ ) was used to detect the presence of apoptotic cells. DVDMS was used at a concentration of $0.5 \mu \mathrm{M}$. After treatment for $6 \mathrm{~h}$, the cells were washed twice with ice-cold PBS and resuspended in $1 \mathrm{X}$ binding buffer at a concentration of $1 \times 10^{6} \mathrm{cells} / \mathrm{ml}$. A total of $5 \mu \mathrm{l} \mathrm{PE}$ Annexin V and $5 \mu \mathrm{l}$ 7-AAD were added per $1 \mathrm{ml}$ cell suspension, and the cells were incubated in the dark at room temperature for $15 \mathrm{~min}$. Apoptotic cells were subsequently identified using a flow cytometer. NAC (cat. no. HY-B0215, Med Chem Express Inc.), a reactive oxygen species (ROS) scavenger (22), was used to observe the effects of ROS on cell death. A total of $1 \mu \mathrm{l} \mathrm{NAC}$ solution $(1,000 \mathrm{mM})$ was added to $1 \mathrm{ml}$ cell culture medium, at a final concentration of $1 \mathrm{mM}$, prior to laser irradiation.

Evaluation of the antitumor effect in vivo. U-118 MG cells $\left(3 \times 10^{7}\right.$ cells $\left./ \mathrm{ml}\right)$ were suspended in PBS containing 50\% Matrigel (BD Biocoat ${ }^{\mathrm{TM}}$ ) and subcutaneously implanted into 4 -week-old BALB/c mice as previously described $(23,24)$. Forty-eight mice were divided into eight groups (6 in each group): The control, laser, ultrasound, DVDMS $(2.0 \mathrm{mg} / \mathrm{kg})$, low-dose DVDMS-PDT (PDT-L,DVDMS $1.0 \mathrm{mg} / \mathrm{kg}$ ), low-dose DVDMS-SDT (SDT-L, DVDMS $1.0 \mathrm{mg} / \mathrm{kg}$ ), high-dose DVDMS-PDT (PDT-H, DVDMS $2.0 \mathrm{mg} / \mathrm{kg}$ ), and high-dose DVDMS-SDT (SDT-H, DVDMS $2.0 \mathrm{mg} / \mathrm{kg}$ ) groups. At $24 \mathrm{~h}$ post DVDMS injection, mice in the laser, PDT-L and PDT-H groups were exposed to the PDT-630 Laser Unit at a luminous flux of $150 \mathrm{~mW} / \mathrm{cm}^{2}$ for $10 \mathrm{~min}$ (total energy, $90.0 \mathrm{~J} / \mathrm{cm}^{2}$ ), while those in the ultrasound, SDT-L and SDT-H groups received ultrasonic irradiation at a frequency of $1.00 \mathrm{MHz}$ and intensity of $500 \mathrm{~mW} / \mathrm{cm}^{2}$ for $3 \mathrm{~min}$ (total energy, $90 \mathrm{~J} / \mathrm{cm}^{2}$ ).

The IVIS ${ }^{\circledR}$ Lumina LT in vivo Imaging system (Series III; Caliper Life Sciences) was used to evaluate the in vivo fluorescence of DVDMS after treatment. The Vevo 2100 Imaging system (FUJIFILM Visual Sonics Inc.) was used to measure the tumor sizes and evaluate the therapeutic effects. Mice were anesthetized using isoflurane (inhalation anesthesia, the induction and maintenance dose was 3 and $1.5 \%$, respectively) before the PDT/SDT treatment, measurements and imaging observations to minimize suffering and distress. Treatment was performed twice (once per week). Two weeks later, when the treatment was over, the mice were euthanized using pentobarbital sodium
$(120 \mathrm{mg} / \mathrm{kg}$, intraperitoneal injection) and the tumors were collected. The sizes and weights of the tumors were measured and H\&E staining was performed to show the significant changes in vascular obstruction and cell apoptosis in the PDT and SDT groups.

Effects of DVDMS-mediated PDT and SDT on protein expression and phosphorylation. Western blot analysis was used to detect protein expression and phosphorylation levels. Briefly, U-118 MG cells were inoculated in 6-well plates $(1 \mathrm{ml} /$ well) for $24 \mathrm{~h}$. Cells in the DVDMS, PDT and SDT groups were treated with DVDMS $(0,0.03,0.13$ and $0.5 \mu \mathrm{M})$ for $4 \mathrm{~h}$. The cells were subsequently treated as aforementioned. At $6 \mathrm{~h}$ after treatment, the cells were collected, washed three times with ice-cold PBS, and lysed with lysis buffer ( $80 \mu \mathrm{l} /$ well; Thermo Fisher Scientific, Inc.) containing $1 \mathrm{mM}$ phenyl methane sulfonyl fluoride (Thermo Fisher Scientific, Inc.), $1 \mathrm{mM} \mathrm{Na} \mathrm{VO}_{4}$ (Sigma-Aldrich; Merck $\mathrm{KGaA}$ ), and $20 \mathrm{mM} \mathrm{NaF}$ (Sigma-Aldrich; Merck KGaA). The mixture was incubated at $4^{\circ} \mathrm{C}$ for $30 \mathrm{~min}$ and then centrifuged at $12,900 \mathrm{x}$ g for $30 \mathrm{~min}$. Protein concentrations were measured using a micro-spectrophotometer and adjusted to $15.00 \mu \mathrm{g} / \mathrm{ml}$. Then, $10.00 \mu \mathrm{l}$ aliquots of the samples were loading into the wells of polyacrylamide gels (spacer gel, 6\%; separation gel, $12 \%$ ) and separated by electrophoresis at $80-120 \mathrm{~V}$, with a constant current of $330 \mathrm{~mA}$ for $90 \mathrm{~min}$. The separated proteins were transferred onto PVDF membranes $(0.22 \mu \mathrm{m})$, which were blocked for $1 \mathrm{~h}$ at room temperature. The membranes were washed three times with Tris-buffered saline containing Tween (10 min per wash), and incubated with primary antibodies overnight at $4^{\circ} \mathrm{C}$ (dilution 1:1,000; PCNA, cat. no. PA5-27214; Bcl-xL, cat. no. PA5-104974; Bax, cat. no. MA5-32031; p-PI3K, cat. no. PA5-104853; p-AKT, cat. no. PA5-95669; p-mTOR, cat. no. 44-1125G; p-p70s6K, cat. no. PA5-104841; p-rps6, cat. no. A300-584A; p-4EBP-1, cat. no. 700238; p-eIF4E, cat. no. 44-528G; p62, cat. no. PA5-20839; LC3, cat. no. PA1-16931; Invitrogen; Thermo Fisher Scientific, Inc.) and then horseradish peroxidase (HRP)-labeled goat anti-rabbit IgG $1 \mathrm{~h}$ at room temperature (dilution 1:5,000; cat. no. 31460; Invitrogen; Thermo Fisher Scientific, Inc.). The protein bands were detected using a chemiluminescence detector (Tanon 5220S; Guangzhou Ewell Bio-technology Co., Ltd.). The gray values of the protein bands were measured using the built-in analysis software of the chemiluminescence detector, and all normalized to the housekeeper control, for phosphorylated antibodies, the protein bands were then normalized to the corresponding unphosphorylated ones. NAC was used to observe the effects of ROS on protein expression and phosphorylation changes induced by DVDMS-mediated PDT and SDT.

Statistical analysis. Statistical analyses were performed using SPSS software (version 23.0; IBM Corp.). Data are expressed as the means \pm standard deviation. The one-way analysis of variance (Tukey's, Dunnett's) was used to compare inter-group differences. $\mathrm{P}<0.05$ was considered to indicate a statistically significant difference.

\section{Results}

DVDMS uptake by U-118 MG cells. As shown in Fig. 1A, five absorption peaks at 369, 517, 550, 577 and $631 \mathrm{~nm}$, 

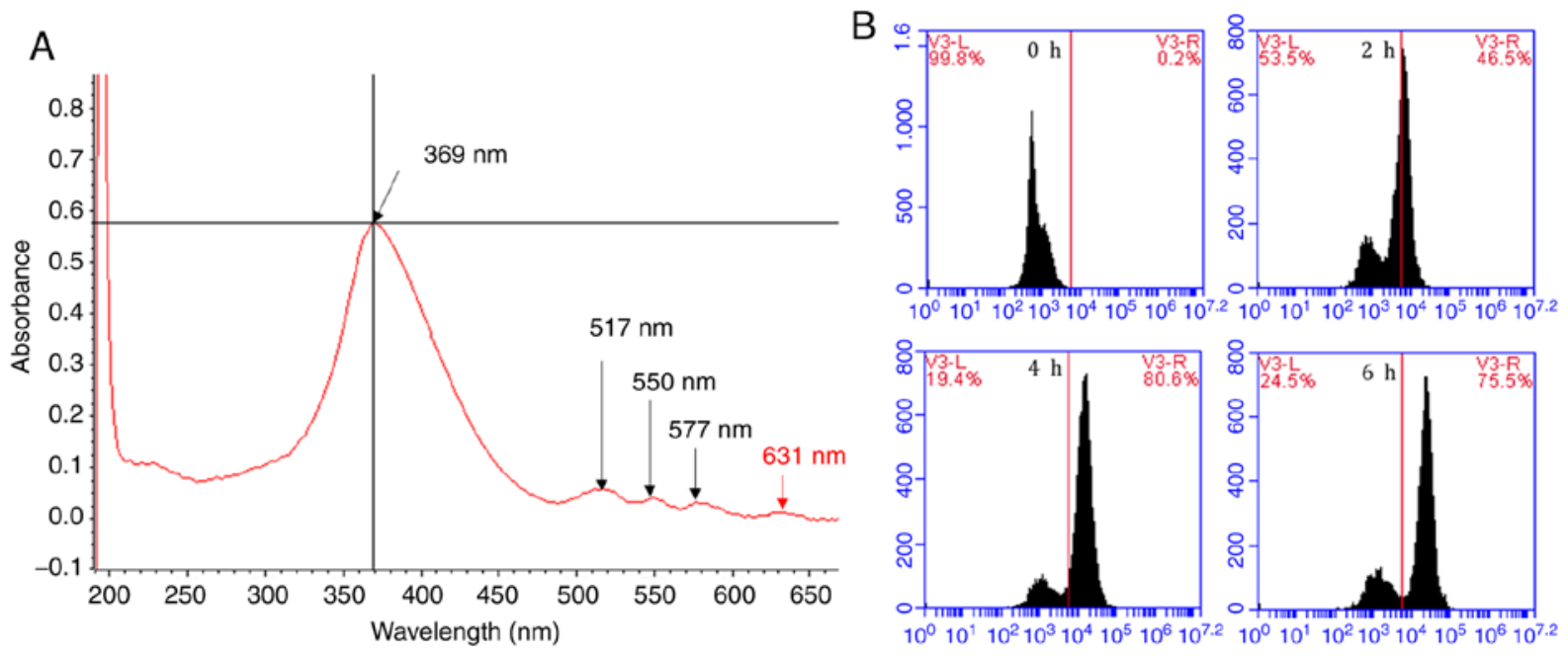

Figure 1. Uptake of DVDMS by U-118 MG cells. (A) The absorption spectrum of DVDMS showed five absorption peaks at 369, 517, 550, 577 and 631 nm, respectively. (B) Cellular uptake after different incubation times $(0,2,4$ and $6 \mathrm{~h})$. The cell density of U-118MG was $10^{6}$ cells $/ \mathrm{ml}$ and the concentration of DVDMS was $8 \mu \mathrm{M}$. Uptake was measured using the APC channel (with excitation and emission wavelengths of $633 \mathrm{and} 660 \mathrm{~nm}$, respectively). DVDMS, sinoporphyrin sodium.

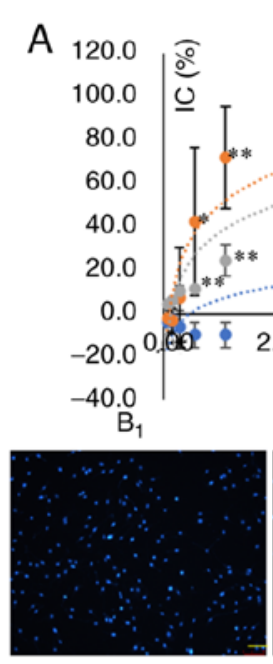

$\mathrm{D}_{1}$
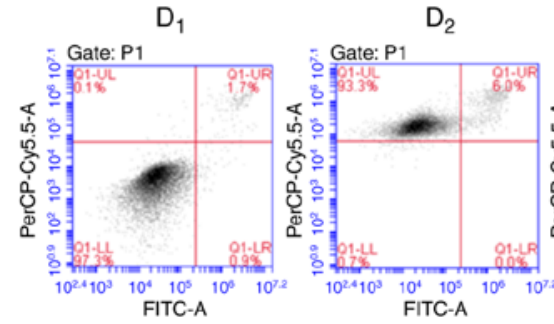

$\bullet$ DVDMS $\quad$ PDT $\quad$ SDT
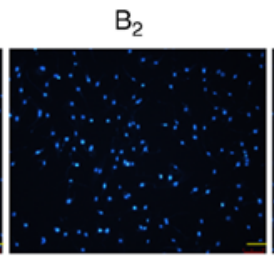

$\mathrm{D}_{2}$

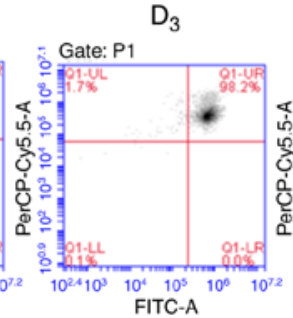

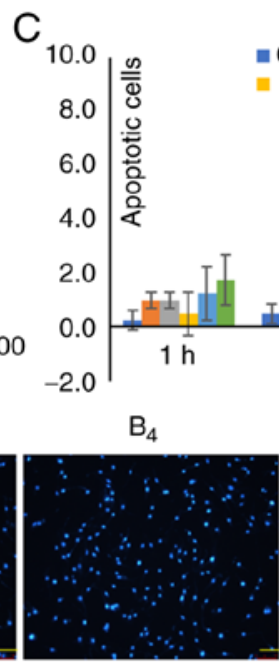

$\mathrm{D}_{4}$

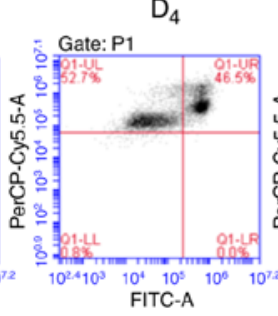

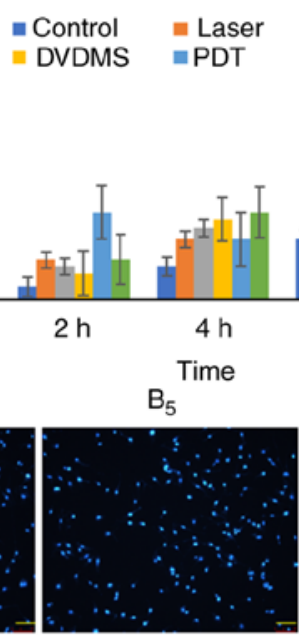

$D_{5}$

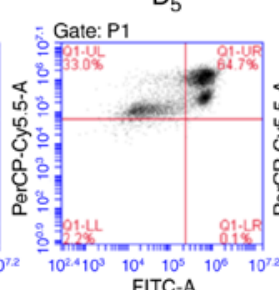

FITC-A
= Ultrasound

= $\mathrm{SDT} \quad * * * *$
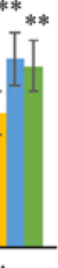

$8 \mathrm{~h}$

$24 \mathrm{~h}$

Figure 2. Effects of DVDMS-mediated PDT and SDT on proliferation and apoptosis of U-118 MG cells. (A) Inhibition of cell proliferation, ${ }^{*} \mathrm{P}<0.05,{ }^{* *} \mathrm{P}<0.01$, ${ }^{* * * *} \mathrm{P}<0.001$ vs. DVDMS. (B) Hoechst 33258 staining of apoptotic cells: $\left(\mathrm{B}_{1}\right)$ control group, $\left(\mathrm{B}_{2}\right)$ laser group, $\left(\mathrm{B}_{3}\right)$ ultrasound group, $\left(\mathrm{B}_{4}\right)$ DVDMS group, $\left(B_{5}\right)$ PDT group, $\left(B_{6}\right)$ SDT group; original magnification was $x 100$, and the yellow bar indicates $100 \mu \mathrm{m}$. (C) Effects of different treatment times on apoptosis, ${ }^{* *} \mathrm{P}<0.01$ vs. the control group. (D) Effects of NAC on PDT- and SDT-induced cell apoptosis: $\left(\mathrm{D}_{1}\right)$ control group, $\left(\mathrm{D}_{2}\right)$ DVDMS group, $\left(\mathrm{D}_{3}\right)$ PDT group, $\left(\mathrm{D}_{4}\right)$ PDT+NAC group, $\left(\mathrm{D}_{5}\right)$ SDT group, $\left(\mathrm{D}_{6}\right)$ SDT+NAC group. DVDMS, sinoporphyrin sodium; PDT, photodynamic therapy; SDT, sonodynamic therapy; NAC, N-acetyl-L-cysteine.

respectively were observed for DVDMS in PBS. The cellular uptake of DVDMS increased with the incubation time. Approximately 50\% of the U-118 MG cells contained DVDMS $2 \mathrm{~h}$ after incubation, which increased to $80 \%$ at $4 \mathrm{~h}$. Subsequently, the cellular uptake began to decrease to $\sim 5 \%$ at $6 \mathrm{~h}$ (Fig. 1B). These results suggested that the optimum uptake of DVDMS by U-118 MG cells occurred at an incubation time of $4 \mathrm{~h}$.

DVDMS-mediated PDT and SDT inhibit the proliferation of glioma cells and induce apoptosis. The CCK-8 assay showed that DVDMS-mediated PDT and SDT inhibited the proliferation of 
A

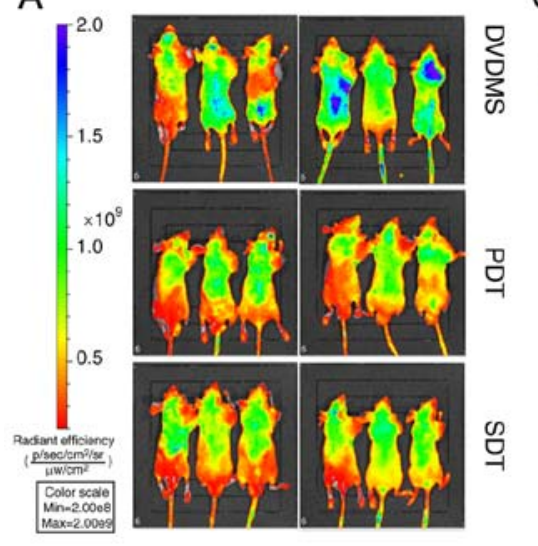

C

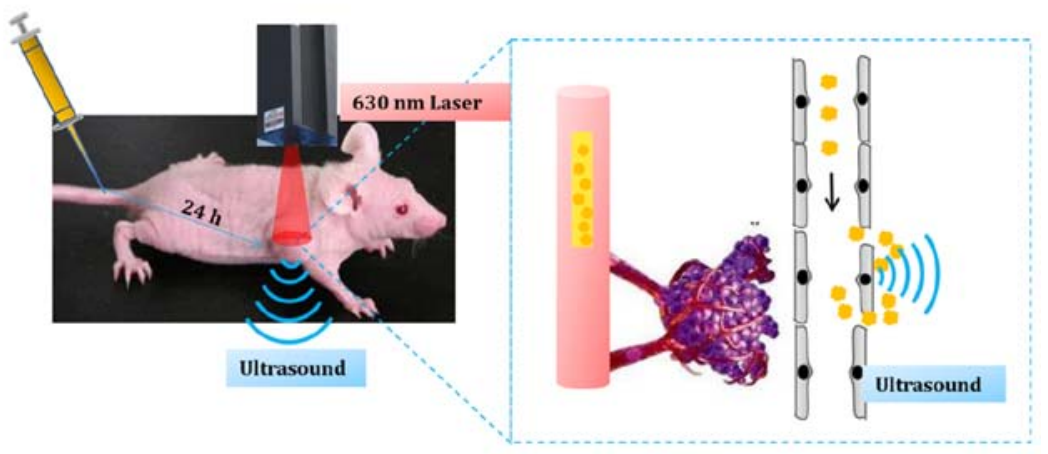

B
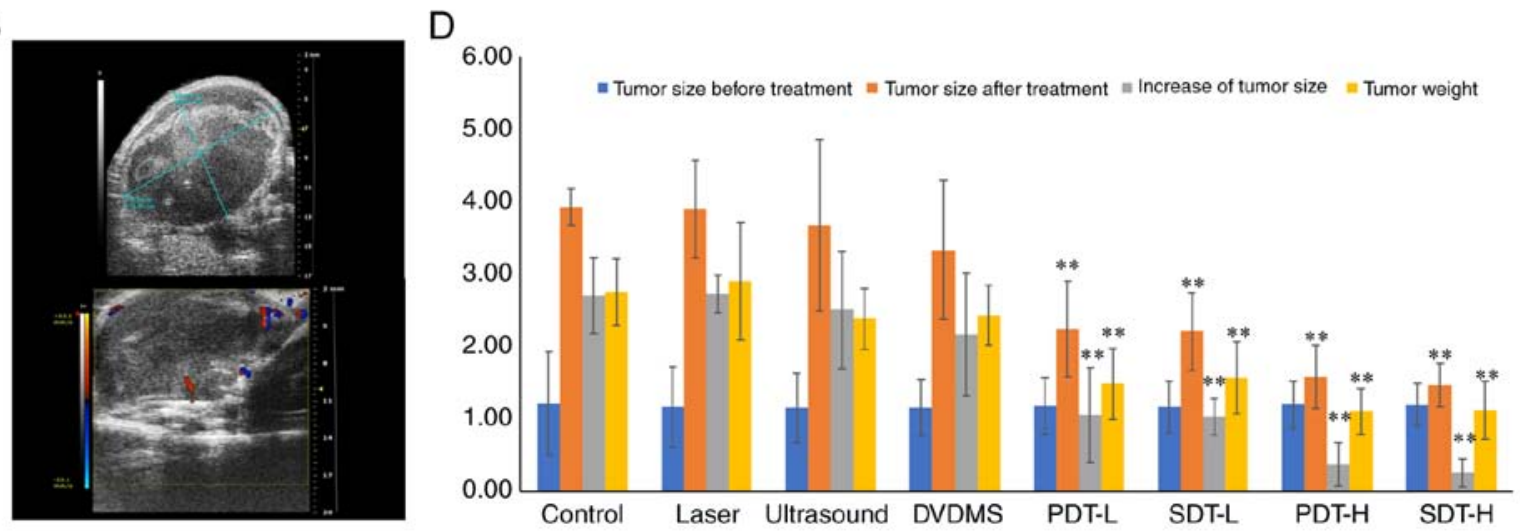

Figure 3. In vivo antitumor effects of DVDMS-mediated PDT and SDT in a xenograft mouse model. (A) In vivo fluorescence measurement. The DVDMS dose in the three groups was $2.00 \mathrm{mg} / \mathrm{kg}$. (B) Representative ultrasound images of a xenograft tumor. (C) Ideograph of DVDMS-mediated PDT and SDT. (D) Comparison of tumor size $\left(\mathrm{cm}^{2}\right)$ and weight (g). The DVDMS dose in the low-dose groups and high-dose groups was 1.0 and $2.0 \mathrm{mg} / \mathrm{kg}$, respectively. PDT was performed using a $630-\mathrm{nm}$ laser with a luminous flux of $150 \mathrm{~mW} / \mathrm{cm}^{2}$ for $10 \mathrm{~min}$. SDT groups received ultrasonic irradiation at $1.00 \mathrm{MHz}$ with an intensity of $500 \mathrm{~mW} / \mathrm{cm}^{2}$ for $3 \mathrm{~min} .{ }^{* *} \mathrm{P}<0.01 \mathrm{vs}$. the control group. DVDMS, sinoporphyrin sodium; PDT, photodynamic therapy; SDT, sonodynamic therapy.

U-118 MG cells (Fig. 2A) and U-87 MG cells (Fig. S1A). Hoechst 33258 staining showed that, the proportion of apoptotic cells increased with increasing time after PDT or SDT (Fig. 2B and C). Annexin V-positive cells were considered to be apoptotic. PDT and SDT increased the number of apoptotic cells compared with the control, and NAC partially abrogated this effect (Fig. 2D).

DVDMS-mediated PDT and SDT inhibit tumor development in $U-118 M G$ xenograft models. The duration of the animal experiment lasted for 6 weeks, including 4 weeks of tumor cell inoculation and 2 weeks PDT and SDT treatment period. There was no mouse found dead during the whole experiment cycle. All mice were euthanized using pentobarbital sodium $(120 \mathrm{mg} / \mathrm{kg})$ when the experiment was over avoiding the pain inducing by tumor growth. The death of mice was verified as follows: The breath, heartbeat and circulation were permanently stopped and the onset of rigor mortis was confirmed. According to previous studies, the maximum concentration of DVDMS in tumors occurs at $24 \mathrm{~h}$ after intravenous injection through the tail vein $(10,11)$. Therefore, PDT and SDT were performed at this time in the present study (Fig. 3C). Ultrasound results in sonoporation, which increases cell clearance and membrane permeability, and may increase delivery of drugs into tumor cells $(25,26)$ (Fig. 3C). In vivo fluorescence revealed that the fluorescence intensity of DVDMS was reduced in the PDT and
SDT groups compared with the DVDMS group, indicating that photobleaching occurred after PDT and SDT (Fig. 3A). DVDMS-PDT and DVDMS-SDT produced biological effects in the tumors. Ultrasound images were taken using the Vevo 2100 Imaging system, which showed that after 4 weeks of tumor cell inoculation, low-echo masses were characteristically elliptical in shape with clear boundaries. The shapes of certain tumors were irregular with uneven internal echoes. Color Doppler flow imaging of peripheral and blood flow signals is presented in Fig. 3B. These findings indicated that ultrasound images could be used as an index to evaluate therapeutic effects. There were no significant differences in the tumor sizes among all eight groups before treatment. However, after treatment, the tumor size of the PDT and SDT groups was significantly decreased compared with the control group $(\mathrm{P}<0.01)$. Tumor cell proliferation and tumor weight were significantly decreased in the PDT and SDT groups compared with the control group $(\mathrm{P}<0.01)$. Individual application of the laser, ultrasound or DVDMS had no therapeutic effects compared with the control group (Fig. 3D). H\&E staining suggested that PDT and SDT induced significant apoptosis and vascular obstruction of the cancer tissue (Fig. S1B).

Effects of DVDMS-mediated PDT and SDT on protein expression and phosphorylation. Western blotting results 

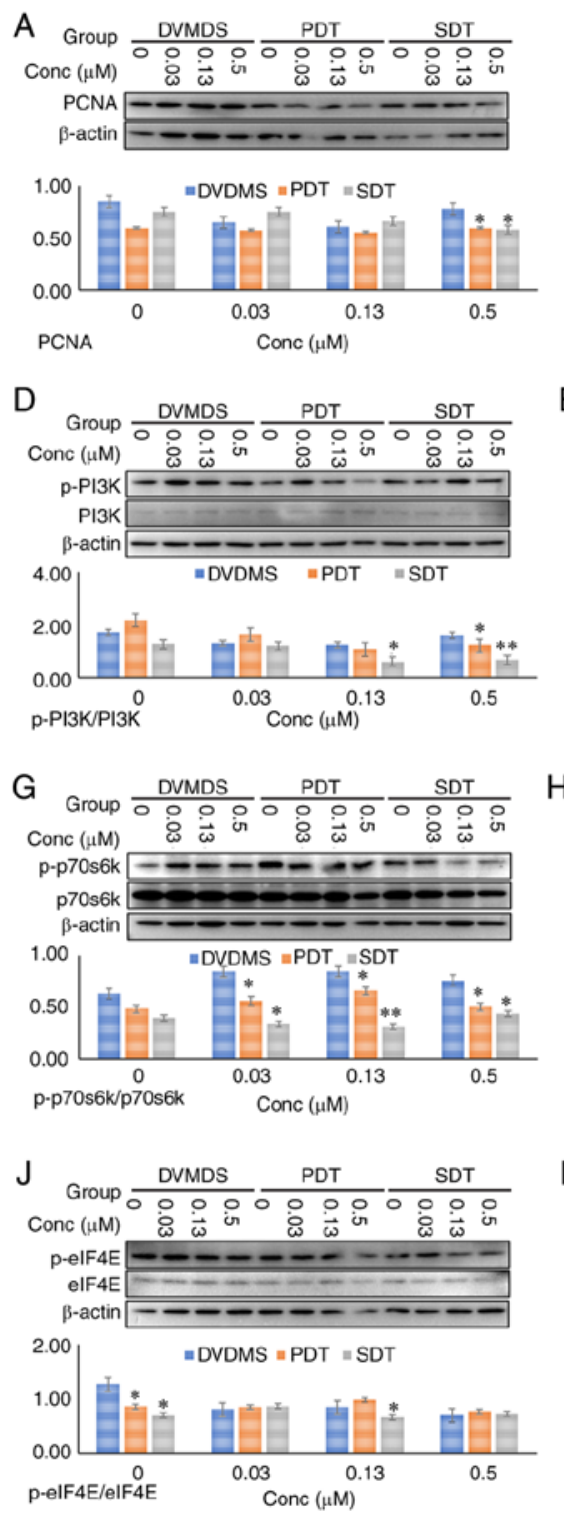
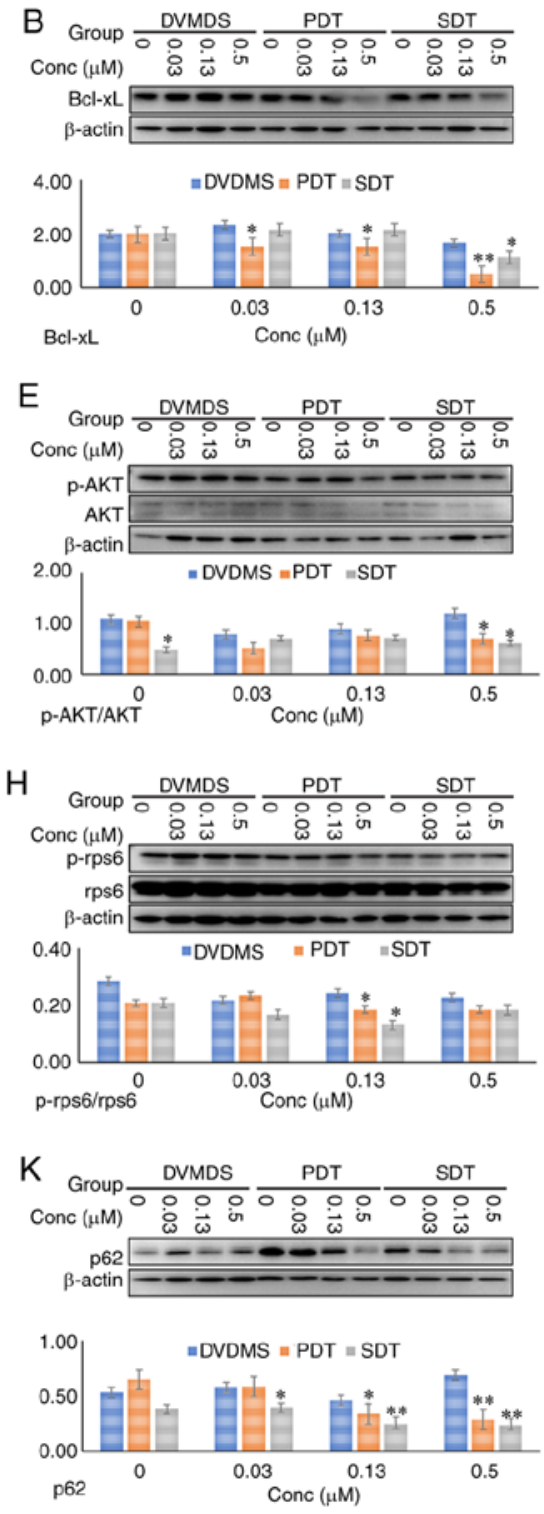
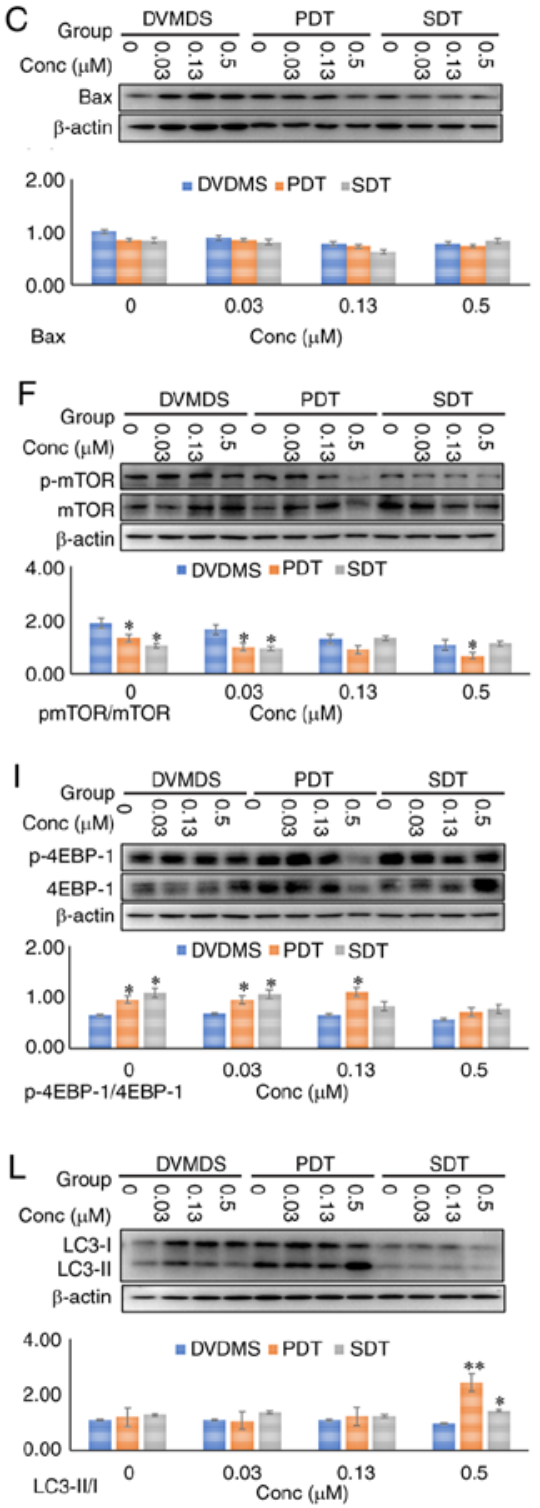

Figure 4. DVDMS-mediated PDT and SDT inhibition of the PI3K/AKT/mTOR signaling pathway. (A) PCNA, (B) Bcl-xL, (C) Bax, (D) p-PI3K, (E) p-AKT, (F) p-mTOR, (G) p-p70s6K, (H) p-rps6, (I) p-4EBP-1, (J) p-eIF4E, (K) p62, (L) LC3-II/LC3-I. Concentrations of DVDMS in the three groups were 0, 0.03, 0.13 and $0.5 \mu \mathrm{M}$. PDT and SDT were performed $4 \mathrm{~h}$ after co-incubation, $\mathrm{n}=3,{ }^{*} \mathrm{P}<0.05,{ }^{* *} \mathrm{P}<0.01$ vs. DVDMS. DVDMS, sinoporphyrin sodium; PDT, photodynamic therapy; SDT, sonodynamic therapy; PCNA, proliferating cell nuclear antigen; Bcl-xL, B-cell lymphoma-extra large; Bax, BCL2-associated X; p-PI3K, phospho-phosphatidylinositol 3 kinase; p-AKT, phospho-protein kinase B; p-mTOR, phospho-mammalian target of rapamycin; p-p70s6k, phospho-p70 ribosomal s6 kinase; p-rps6, phospho-ribosomal protein s6; p-4EBP1, phospho-4E binding protein 1; p-eIF4E, phospho-protein synthesis initiation factor 4E; SQSTM1/p62, sequestosome 1; LC3, light chain 3.

showed that PDT and SDT enhanced DVDMS suppression of PCNA and Bcl-xL, however, the levels of Bax were not significantly different in the 3 groups (Fig. 4A-C). PCNA is a member of the DNA sliding clamp family of proteins that are involved in DNA replication, repair and the cell cycle. PCNA protein expression is a well-accepted marker of proliferation (27). Suppression of PCNA may inhibit glioma cells in vitro. $\mathrm{Bcl}-\mathrm{xL}$ is a $30-\mathrm{kDa}$ anti-apoptotic protein that belong to the $\mathrm{Bcl}-2$ family, and it can prevent apoptosis through two different mechanisms: i) Heterodimerization with an apoptotic protein, thereby inhibiting the apoptotic effect; and ii) formation of mitochondrial outer membrane pores that help maintain a normal membrane state under stressful conditions (28). Therefore, suppression of Bcl-xL by DVDMS-mediated PDT and SDT may lead to cell apoptosis.
Furthermore, the suppression of PCNA and Bcl-xL induced by PDT and SDT was partly reversed by NAC, as well as the enhanced cleaved-caspase 3 levels (Fig. S1C). These results indicated that ROS was the main factor for DVDMS-mediated PDT and SDT. This supported the flow cytometry results demonstrating that NAC reduced PDT and SDT-induced apoptosis in vitro.

The phosphorylation cascades of the PI3K/AKT/mTOR signaling pathway can be suppressed by ROS to induce autophagy (29). The results showed that the phosphorylation levels of PI3K, AKT, mTOR, p70s6K, rps6 and eIF4E were more significantly inhibited by both PDT and SDT, compared with DVDMS alone; however, the phosphorylation levels of 4EBP-1 were significantly increased in the PDT and SDT groups than that in DVDMS group (Fig. 4D-J). p62 and 
LC3 are markers of autophagy. During autophagy, LC3-I is converted to LC3-II through lipidation by a ubiquitin-like system. Lysosomal degradation of autophagosomes leads to a decrease in p62 levels during autophagy; conversely, autophagy inhibitors stabilize p62 levels $(30,31)$. In the present study, both PDT and SDT significantly inhibited the p62 levels (Fig. 4K), however, only PDT promoted the conversion of LC3-I to LC3-II (Fig. 4L).

\section{Discussion}

Sinoporphyrin sodium (DVDMS) is a novel sensitizer that is activated by both laser and ultrasound. Previous studies have confirmed that DVDMS-mediated photodynamic therapy (PDT) and sonodynamic therapy (SDT) are effective against several types of tumors through mechanisms such as ROS generation, DNA damage, matrix metallic proteinase downregulation, collapse of F-actin filaments and cell apoptosis (32-34). The present study demonstrated the antitumor effects of DVDMS-PDT and DVDMS-SDT in $\mathrm{U}-118 \mathrm{MG}$ cells in vitro and in vivo. The results showed that both DVDMS-PDT and DVDMS-SDT significantly inhibited proliferation and induced apoptosis of $\mathrm{U}-118 \mathrm{MG}$ cells in vitro. Moreover, in a nude mouse xenograft model, the fluorescence intensity of DVDMS was lower in both the PDT and SDT groups compared with the DVDMS group, while tumor proliferation and weight were lower in the PDT and SDT groups compared with the control group. These results indicated that both DVDMS-PDT and DVDMS-SDT may serve as potential strategies for glioma treatment. Burns and pain are the most common adverse reactions caused by PDT, resulting in decreased patient compliance. These side effects are mainly caused by the photothermal effects of the laser. In the present study, a low power laser with a long irradiation time showed good antitumor effects, indicating that a low-power laser could be considered in future studies.

The present study had certain limitations. Future studies investigating an orthotopic transplantation model should be used to validated the therapeutic effects observed, as the environment of tumor cells in the cranium may differ to that under the skin. Furthermore, the blood-brain barrier (BBB) may block the entry of DVDMS to the brain, therefore, drug delivery systems, such as microbubbles, should be considered to increase treatment efficiency. Recently, Pi et al (35) used focused ultrasound with microbubbles to open the BBB to enhance the delivery of DVDMS to intracranial U-87 MG tumors, and then used another ultrasound to active DVDMS. The results revealed that DVDMS-SDT significantly inhibited glioma in vivo. Liu et al (36) encapsulated DVDMS chelated with manganese ions into nanoliposomes and demonstrated enhanced antitumor effects, suggesting that this approach may be a promising strategy for glioma treatment. Sun et al (37) used ultrasound-targeted microbubble destruction combined with iRGD-modified DVDMS liposomes to treat glioma, and found that the orthotopically implanted C6 gliomas were significantly suppressed. The aforementioned studies provide potential strategies for the treatment of brain gliomas with DVDMS-SDT.

As the laser will not penetrate the skull, DVDMS-PDT may be more challenging than SDT. However, there may be some potential uses of DVDMS and its light sensitivity. Since glioma is an invasive growth with unclear boundaries, it is difficult to completely remove by surgery and patients are prone to recurrence. DVDMS can be used as a fluorescent indicator to display the tumor boundaries and guide surgical resection, on the other hand, postoperative DVDMS-PDT may inhibit tumor recurrence. Yan et al (38) developed a novel photo-theranostic agent based on DVDMS-loaded PEGylated graphene oxide with improved fluorescence properties for enhanced optical imaging guided PDT. Photoacoustic imaging (PAI) is a novel non-invasive biomedical imaging method developed in recent years. As DVDMS is activated by both laser and ultrasound, it may be used in PAI. DVDMS and PAI may be used in image-guided therapy for glioma surgery, or to dynamically monitor the therapeutic effect. Furthermore, PDT and SDT may enhance the therapeutic effects. An in vivo PAI system may be used with an orthotopic transplantation model to validate the aforementioned effects.

Previous studies have revealed that DVDMS has good water solubility and stability, is widely applicable in PDT and SDT, and shows good therapeutic effects against tumors, bacteria and skin diseases $(11,39)$. Preclinical safety evaluation showed that DVDMS as a good safety profile in SD rats and beagles $(10,40)$. Furthermore, there are two registered phase I clinical trials investigating DVDMS for the treatment of esophageal cancer and advanced solid tumors (no. CTR20150690 and CTR20150725, respectively). The results of the present study showed that DVDMS-mediated PDT and SDT demonstrated good inhibition of glioma, indicating that DVDMS may be a promising sensitizer for both PDT and SDT.

The majority of the cell-damaging effects induced by PDT and SDT are generally attributed to ROS. Sun et al (41) found that ROS were major triggering intermediates during the DVDMS-SDT treatment of human glioma U-373 MG cells, and that the increase in apoptosis was accompanied by an increase in cleaved-caspase 3 level and DNA fragmentation. Our study indicated that DVDMS-mediated PDT and SDT significantly inhibited the expression levels of PCNA and Bcl-xL, and enhanced cleaved-caspase 3. The ROS scavenger NAC partly reversed these changes, and reduced cell apoptosis induced by DVDMS-mediated PDT and SDT, as demonstrated by the flow cytometry results. These results indicated that ROS may play an important role in inhibition of cell proliferation and induction of cell apoptosis by DVDMS-mediated PDT and SDT.

The PI3K/AKT/mTOR signaling pathway is important in the onset and progression of cancer and it can be regulated by ROS (42). Recent studies have shown that both PDT and SDT inhibit the PI3K/AKT/mTOR signaling pathway. Han et al (43) found that PDT mediated by fluorescent nanoparticles encapsulating Chlorin e6 suppressed p-mTOR and p-AKT via ROS generation in macrophage-derived foam cells. Zheng et al (44) revealed that SDT mediated by hydroxyl acetylated curcumin induced PI3K/AKT/mTOR pathway-dependent autophagy, and the mitochondrial-caspase pathway was crucial in apoptosis. The results of the present study demonstrated that DVDMS-mediated PDT and SDT efficiently inhibited the $\mathrm{PI} 3 \mathrm{~K} / \mathrm{AKT} / \mathrm{mTOR}$ signaling pathway.

The results of the present study revealed that the phosphorylation of p70s6K, rps6 and eIF4E, downstream of mTORC1 
were decreased, and the phosphorylation of 4EBP1 was increased, which is generally thought to be upregulated when this signaling pathway is inhibited (45), and it may therefore require further investigation. Recent studies have revealed that autophagy pathways are activated in response to PDT or SDT. The activation of autophagy pathways was also associated with the PI3K/AKT/mTOR signaling pathway. However, the effects of autophagy activation and antitumor effects in PDT and SDT remain controversial (46-49). In the present study, we found that both DVDMS-PDT and DVDMS-SDT significantly inhibited the p62 levels, however, only PDT promoted the conversion from LC3-I to LC3-II. Interestingly, unlike PCNA, Bcl-xL, and caspase-3, changes in LC3 induced by PDT were not reversed by NAC, whereas in SDT, NAC induced a ignificant increase in LC3II/I (Fig. S1C), highlighting the differences between PDT- and SDT-induced autophagy. The role of autophagy in PDT- and SDT-induced cell damage is very complex. Shi et al (34) found that DVDMS-PDT induced apoptosis and autophagy in esophageal cancer cells via ROS generation, while inhibiting autophagy reduced DVDMS-PDT-triggered apoptosis. However, Zhu et al (50) found that inhibiting autophagy with chloroquine enhanced DVDMS-PDT-induced apoptosis in colorectal cancer cells. To the best of our knowledge, the present study was the first to reveal the influences of DVDMS-SDT on autophagy; however, the association between autophagy and apoptosis remain unclear. Further studies are warranted to explore the mechanisms of autophagy in regulating glioma cell apoptosis induced by DVDMS-mediated PDT and SDT.

\section{Acknowledgements}

We would like to thank Professor Qi-Cheng Fang for kindly providing the DVDMS.

\section{Funding}

This study was financially supported by the Science and Technology Planning Project of Shenzhen (grant no. JCY J20180302153611416), the National Natural Science Fund of China-Henan Joint Fund (grant no. U1804187) and the National Science and Technology Major Projects of China for 'Major New Drugs Innovation Development' (grant no. 2018ZX09201017-005).

\section{Availability of data and materials}

The datasets used and/or analyzed during the current study are available from the corresponding author on reasonable request.

\section{Authors' contributions}

YWA and HQL were the main conductors of the experiments. FW designed the in vivo experiments. HTJ reviewed the data and drafted the manuscript. HQL was responsible for submission and answering to reviewers' comments. GYJ, ZWL and ZQZ conducted the western blotting, PDT and SDT, respectively. JCW carried out the statistics. All authors read and approved the final manuscript.

\section{Ethics approval and consent to participate}

The present study was approved by the Ethics Committee of Shenzhen PKU-HKUST Medical Center (SYXK 2015-0106).

\section{Patient consent for publication}

Not applicable.

\section{Competing interest}

The authors declare that they have no competing interest.

\section{References}

1. Kwiatkowski S, Knap B, Przystupski D, Saczko J, Kędzierska E, Knap-Czop K, Kotlińska J, Michel O, Kotowski K and Kulbacka J: Photodynamic therapy-mechanisms, photosensitizers and combinations. Biomed Pharmacother 106: 1098-1107, 2018.

2. Ang JM, Riaz IB, Kamal MU, Paragh G and Zeitouni NC: Photodynamic therapy and pain: A systematic review. Photodiagnosis Photodyn Ther 19: 308-344, 2017.

3. McHale AP, Callan JF, Nomikou N, Fowley C and Callan B: Sonodynamic therapy: Concept, mechanism and application to cancer treatment. Adv Exp Med Biol 880: 429-450, 2016.

4. Fitzmaurice S and Eisen DB: Daylight photodynamic therapy: What is known and what is yet to be determined. Dermatol Surg 42: 286-295, 2016.

5. Rkein AM and Ozog DM: Photodynamic therapy. Dermatol Clin 32: 415-425, 2014.

6. Chilakamarthi U and Giribabu L: Photodynamic therapy: Past, present and future. Chem Rec 17: 775-802, 2017.

7. Wang X, Jia Y, Wang P, Liu Q and Zheng H: Current status and future perspectives of sonodynamic therapy in glioma treatment. Ultrason Sonochem 37: 592-599, 2017.

8. Abrahamse H and Hamblin MR: New photosensitizers for photodynamic therapy. Biochem J 473: 347-364, 2016.

9. Ibbotson SH: Adverse effects of topical photodynamic therapy. Photodermatol Photoimmunol Photomed 27: 116-130, 2011.

10. Lin N, Li C, Wang Z, Zhang J, Ye X, Gao W, Wang A, Jin H and Wei J: A safety study of a novel photosensitizer, sinoporphyrin sodium, for photodynamic therapy in Beagle dogs. Photochem Photobiol Sci 14: 815-832, 2015.

11. Shi R, Li C, Jiang Z, Li W, Wang A and Wei J: Preclinical study of antineoplastic sinoporphyrin sodium-PDT via in vitro and in vivo models. Molecules 22: 112, 2017.

12. Xiong W, Wang P, Hu J, Jia Y, Wu L, Chen X, Liu Q and Wang X: A new sensitizer DVDMS combined with multiple focused ultrasound treatments: An effective antitumor strategy. Sci Rep 5: 17485, 2015.

13. Liu Y, Wang P, Liu Q and Wang X: Sinoporphyrin sodium triggered sono-photodynamic effects on breast cancer both in vitro and in vivo. Ultrason Sonochem 31: 437-448, 2016.

14. Sun Q, Xu R, Xu H, Wang G, Shen X and Jiang H: Extracranial metastases of high-grade glioma: The clinical characteristics and mechanism. World J Surg Oncol 15: 181, 2017.

15. McGranahan T, Li G and Nagpal S: History and current state of immunotherapy in glioma and brain metastasis. Ther Adv Med Oncol 9: 347-368, 2017.

16. Uhm JH and Porter AB: Treatment of glioma in the 21st century: An exciting decade of postsurgical treatment advances in the molecular era. Mayo Clin Proc 92: 995-1004, 2017.

17. Long W, Yi Y, Chen S, Cao Q, Zhao W and Liu Q: Potential new therapies for pediatric diffuse intrinsic pontine glioma. Front Pharmacol 8: 495, 2017.

18. Zavadskaya TS: Photodynamic therapy in the treatment of glioma. Exp Oncol 37: 234-241, 2015.

19. Noske DP, Wolbers JG and Sterenborg HJ: Photodynamic therapy of malignant glioma. A review of literature. Clin Neurol Neurosurg 93: 293-307, 1991.

20. Hao D, Song Y, Che Z and Liu Q: Calcium overload and in vitro apoptosis of the C6 glioma cells mediated by sonodynamic therapy (hematoporphyrin monomethyl ether and ultrasound). Cell Biochem Biophys 70: 1445-1452, 2014. 
21. Nonaka M, Yamamoto M, Yoshino S, Umemura S, Sasaki K and Fukushima T: Sonodynamic therapy consisting of focused ultrasound and a photosensitizer causes a selective antitumor effect in a rat intracranial glioma model. Anticancer Res 29: 943-950, 2009.

22. Halasi M, Wang M, Chavan TS, Gaponenko V, Hay N and Gartel AL: ROS inhibitor N-acetyl-L-cysteine antagonizes the activity of proteasome inhibitors. Biochem J 454: 201-208, 2013

23. Song Y, Cho G, Suh JY, Lee CK, Kim YR, Kim YJ and Kim JK Dynamic contrast-enhanced MRI for monitoring antiangiogenic treatment: Determination of accurate and reliable perfusion parameters in a longitudinal study of a mouse xenograft model. Korean J Radiol 14: 589-596, 2013.

24. Choi H, Gillespie DL, Berg S, Rice C, Couldwell S, Gu J, Colman H, Jensen RL and Huang LE: Intermittent induction of HIF-1alpha produces lasting effects on malignant progression independent of its continued expression. PLoS One 10: e0125125, 2015.

25. Yu H and Xu L: Cell experimental studies on sonoporation: State of the art and remaining problems. J Control Release 174: 151-160, 2014.

26. Muleki Seya P, Fouqueray M, Ngo J, Poizat A, Inserra C and Béra JC: Sonoporation of adherent cells under regulated ultrasound cavitation conditions. Ultrasound Med Biol 41: 1008-1019, 2015.

27. Goodlad RA: Quantification of epithelial cell proliferation, cell dynamics, and cell kinetics in vivo. Wiley Interdiscip Rev Dev Biol 6, 2017.

28. Levesley J, Steele L, Brüning-Richardson A, Davison A, Zhou J, Ding C, Lawler S and Short SC: Selective BCL-XL inhibition promotes apoptosis in combination with MLN8237 in medulloblastoma and pediatric glioblastoma cells. Neuro Oncol 20 203-214, 2018

29. Chen L, Liu P, Feng X and Ma C: Salidroside suppressing LPS-induced myocardial injury by inhibiting ROS-mediated $\mathrm{PI}$ K/Akt/mTOR pathway in vitro and in vivo. J Cell Mol Med 21: 3178-3189, 2017

30. Lamark T, Svenning S and Johansen T: Regulation of selective autophagy: The p62/SQSTM1 paradigm. Essays Biochem 61 609-624, 2017

31. Jiang P and Mizushima N: LC3- and p62-based biochemical methods for the analysis of autophagy progression in mammalian cells. Methods 75: 13-18, 2015.

32. Hu J, Wang X, Liu Q, Zhang K, Xiong W, Xu C, Wang P and Leung AW: Antitumor effect of sinoporphyrin sodium-mediated photodynamic therapy on human esophageal cancer Eca-109 cells. Photochem Photobiol 90: 1404-1412, 2014.

33. Yao J, Gao W, Wang Y, Wang L, Diabakte K, Li J, Yang J, Jiang Y, Liu Y, Guo S, et al: Sonodynamic therapy suppresses neovascularization in atherosclerotic plaques via macrophage apoptosis-induced endothelial cell apoptosis. JACC Basic Transl Sci 5: 53-65, 2019

34. Shi Y, Zhang B, Feng X, Qu F, Wang S, Wu L, Wang X, Liu Q, Wang $\mathrm{P}$ and Zhang $\mathrm{K}$ : Apoptosis and autophagy induced by DVDMs-PDT on human esophageal cancer Eca-109 cells. Photodiagn Photodyn Ther 24: 198-205, 2018.

35. Pi Z, Huang Y, Shen Y, Zeng X, Hu Y, Chen T, Li C, Yu H, Chen S and Chen X: Sonodynamic therapy on intracranial glioblastoma xenografts using sinoporphyrin sodium delivered by ultrasound with microbubbles. Ann Biomed Eng 47: 549-562, 2019.

36. Liu H, Zhou M, Sheng Z, Chen Y, Yeh CK, Chen W, Liu J, Liu X, Yan $\mathrm{F}$ and Zheng H: Theranostic nanosensitizers for highly efficient MR/fluorescence imaging-guided sonodynamic therapy of gliomas. J Cell Mol Med 22: 5394-5405, 2018.
37. Sun $\mathrm{Y}$, Wang H, Wang P, Zhang K, Geng X, Liu Q and Wang X: Tumor targeting DVDMS-nanoliposomes for an enhanced sonodynamic therapy of gliomas. Biomater Sci 7: 985-994, 2019.

38. Yan X, Niu G, Lin J, Jin AJ, Hu H, Tang Y, Zhang Y, Wu A, Lu J, Zhang S, et al: Enhanced fluorescence imaging guided photodynamic therapy of sinoporphyrin sodium loaded graphene oxide. Biomaterials 42: 94-102, 2015.

39. Mai B, Wang X, Liu Q, Leung AW, Wang X, Xu C and Wang P. The antibacterial effect of sinoporphyrin sodium photodynamic therapy on Staphylococcus aureus planktonic and biofilm cultures. Lasers Surg Med 48: 400-408, 2016.

40. Shi R, Lin X, Zhang J, Jin H, Wang A and Wei J: Safety evaluation of repeated intravenous infusion of sinoporphyrin with and without PDT in rats. Photochem Photobiol Sci 15: 1366-1376, 2016.

41. Sun Y, Wang H, Zhang K, Liu J, Wang P, Wang X and Liu Q: Sonodynamic therapy induces oxidative stress, DNA damage and apoptosis in glioma cells. RSC Adv 8: 36245-36256, 2018.

42. Wang X, Fu YF, Liu X, Feng G, Xiong D, Mu GF and Chen FP: ROS promote Ox-LDL-induced platelet activation by up-regulating autophagy through the inhibition of the PI3K/AKT/mTOR pathway. Cell Physiol Biochem 50: 1779-1793, 2018.

43. Han XB, Li HX, Jiang YQ, Wang H, Li XS, Kou JY, Zheng YH, Liu ZN, Li H, Li J, et al: Upconversion nanoparticle-mediated photodynamic therapy induces autophagy and cholesterol efflux of macrophage-derived foam cells via ROS generation. Cell Death Dis 8: e2864, 2017.

44. Zheng L, Li Y, Li X, Kou J, Zhong Z, Jiang Y, Liu Z, Tian Y and Yang L: Combination of hydroxyl acetylated curcumin and ultrasound induces macrophage autophagy with anti-apoptotic and anti-lipid aggregation effects. Cell Physiol Biochem 39: 1746-1760, 2016

45. Huang CI, Wang CC, Tai TS, Hwang TZ, Yang CC, Hsu CM and Su YC: eIF4E and 4EBP1 are prognostic markers of head and neck squamous cell carcinoma recurrence after definitive surgery and adjuvant radiotherapy. PLoS One 14: e0225537, 2019.

46. Reiners JJ Jr, Agostinis P, Berg K, Oleinick NL and Kessel D: Assessing autophagy in the context of photodynamic therapy. Autophagy 6: 7-18, 2010.

47. Xiong L, Liu Z, Ouyang G, Lin L, Huang H, Kang H, Chen W, Miao X and Wen Y: Autophagy inhibition enhances photocytotoxicity of Photosan-II in human colorectal cancer cells. Oncotarget 8: 6419-6432, 2017.

48. Dewaele M, Martinet W, Rubio N, Verfaillie T, de Witte PA, Piette $\mathrm{J}$ and Agostinis P: Autophagy pathways activated in response to PDT contribute to cell resistance against ROS damage. J Cell Mol Med 15: 1402-1414, 2011.

49. Li X, Zhang X, Zheng L, Kou J, Zhong Z, Jiang Y, Wang W, Dong Z, Liu Z, Han X, et al: Hypericin-mediated sonodynamic therapy induces autophagy and decreases lipids in THP-1 macrophage by promoting ROS-dependent nuclear translocation of TFEB. Cell Death Dis 7: e2527, 2016.

50. Zhu B, Li S, Yu L, Hu W, Sheng D, Hou J, Zhao N, Hou X, Wu Y, Han Z, et al: Inhibition of autophagy with chloroquine enhanced sinoporphyrin sodium mediated photodynamic therapy-induced apoptosis in human colorectal cancer cells. Int J Biol Sci 15 12-23, 2019.

This work is licensed under a Creative Commons Attribution-NonCommercial-NoDerivatives 4.0 International (CC BY-NC-ND 4.0) License. 